\title{
Unified framework to evaluate non-visual spectral effectiveness of light for human health
}

\author{
Maria L Amundadottir ${ }^{\mathrm{a}}$, Steven W Lockley ${ }^{\mathrm{b}, \mathrm{c}}$ and Marilyne Andersen ${ }^{\mathrm{a}}$ \\ ${ }^{a}$ Interdisciplinary Laboratory of Performance-Integrated Design, École Polytechnique \\ Fédérale de Lausanne, Lausanne, $\mathrm{CH}$ \\ ${ }^{\mathrm{b}}$ Division of Sleep and Circadian Disorders, Department of Medicine, Brigham and Women's \\ Hospital, Boston, MA, USA \\ ${ }^{\mathrm{c}}$ Division of Sleep Medicine, Harvard Medical School, Boston, MA, USA
}

Received December 9, 2015; Revision received May 19, 2016; Accepted May 31, 2016

Published online before print June 27, 2016, doi: 10.1177/1477153516655844

\section{Abstract}

The discovery of a novel non-rod, non-cone photoreceptor in the mammalian eye that mediates a range of 'non-visual' responses to light has required reexamination of how lighting needs for human health are characterized and evaluated. Existing literature provides useful information about how to quantify non-visual spectral sensitivities to light but the optimal approach is far from decided. As more is learned about the underlying biology, new approaches will continue to be published. What is currently lacking is a flexible framework to describe the non-visual spectral effectiveness of light using a common language. Without a unified description of quantities and units, much of the value of scientific publications can be lost. In this paper, we review the existing approaches by categorizing the proposed quantities depending on their application. Based on this review, a unified framework is provided for use in evaluating and reporting the spectral effectiveness of light for human health. The unified framework will provide greater flexibility to model the non-visual responses to light and is adaptable to a wide range of lighting solutions of interest for researchers, designers and developers. A new visualization tool, the SpeKtro dashboard, is available to explore the unified framework on-line at spektro.epfl.ch.

Keywords: Light, health, wavelength, lighting design, light quantities, non-visual, circadian, spectrum 


\section{Introduction}

Ocular light exposure stimulates not only visual functions but also a range of non-visual effects. Studies on humans have demonstrated that both monochromatic short-wavelength light and blue-enriched polychromatic light are more effective than light at longer wavelengths at suppressing melatonin, ${ }^{1-3}$ resetting the circadian clock, ${ }^{4,5}$ enhancing alerting effects and cognitive function, ${ }^{3,6-8}$ and constricting the pupil. ${ }^{9,10}$ These non-visual effects of light are primarily mediated by recently discovered intrinsically photosensitive retinal ganglion cells (ipRGCs). ${ }^{11}$ These cells use melanopsin as a photopigment and, as a result, the ipRGCs are characterized by a spectral sensitivity curve that peaks in the short wavelength region around $490 \mathrm{~nm}$, estimated in vivo, distinguished from the spectral sensitivity of rod and cone photoreceptors. ${ }^{12,13}$

Before the discovery of the new photoreceptor, properties of light exposure were often reported in terms of illuminance. Illuminance is not appropriate to evaluate the non-visual spectral effectiveness of ocular light exposure, because of the different spectral sensitivities of the visual and non-visual systems (555 $\mathrm{nm}$ and $490 \mathrm{~nm}$, respectively). Despite this limitation, illuminance is still widely used in lighting practice to quantify the brightness of a space and its stimulus on the visual system. Recently, new and revised light quantities have been proposed that use traditional approaches consistent with current standards developed to describe light for visual function by the International Commission on Illumination (CIE) $)^{13,14}$ or define light based on the relative relationship between the spectral sensitivity of the nonvisual system and the photopic visual system. ${ }^{15-17}$ While providing useful information about how to evaluate and report non-visual spectral sensitivity to light, these differing approaches are not necessarily interchangeable. A need for a common language of evaluating and reporting the potential non-visual responses of different light is growing within both the academic and lighting design and manufacturing communities. This problem has been recognized ${ }^{17-19}$ and international committees were established to minimize the confusion produced by inconsistent technical terminology. It also persists in related fields of photobiological science that extend beyond human vision, where Sliney ${ }^{20}$ argues that there is no agreement between scientific communities on how to follow standardized terminology to report the potential of light to induce biological responses.

New light quantities and units have been developed by different disciplines driven by different goals to describe similar phenomena and therefore the terms are inconsistent. A next logical step would be to integrate the multiple approaches and construct a more 
comprehensive and unified description while fulfilling the needs of various users. A unified framework might be universally acceptable and would allow information to be exchanged between users and fields of use. Once available, it should help researchers to evaluate and report light source spectral properties consistently, and facilitate understanding of how the five human ocular photoreceptors combine to induce biological responses and ultimately to support lighting design. ${ }^{17}$

In the current paper, the relationships between light quantities for visual function standardized by the CIE and more recent quantities for non-visual spectral sensitivity to light are categorized and explained to provide an adequate basis for explaining the structure and the formulation of the unified framework. It was developed around a new unit-less factor, the relative spectral effectiveness (RSE) factor, which is computed in relation to either spectral energy or luminous-weighted spectrum. ${ }^{21}$ The unified framework provides an improved set of quantities and naming conventions to increase the flexibility of current recommendations ${ }^{17-19}$ for describing non-visual spectral effectiveness of light. The main goal is to help the investigator to develop a conceptual understanding of the various ways of evaluating spectral effectiveness of ocular light exposure using the RSE factor and gain sufficient knowledge to support its usage. A new visualization tool, the SpeKtro dashboard, is available to explore the unified framework on-line at spektro.epfl.ch, where it is possible to upload and analyze spectral distribution of any light source. The application of the framework spans a wide range of interests and is demonstrated using four hypothetical case studies.

\section{Background}

Visual and non-visual responses begin with the detection of light by photoreceptors in the eye. In order to derive a quantitative measure, it is necessary to characterize the human response by the incorporation of a weighting function. A wide selection of spectral weighting functions exists in the literature. The most relevant ones are explained and discussed in Section 2.1. In Section 2.2, the CIE standard for deriving light quantities for photopic and scotopic vision is described in relation to more recent approaches that have been proposed to quantify the spectral sensitivity of the non-visual system to light.

\subsection{Spectral weighting functions}

A spectral weighting function, an action spectrum, represents the relative spectral effectiveness of light required to induce a certain biologic response in a specified system. ${ }^{22}$ The response of the standard light adapted eye for photopic vision is denoted by the spectral 
luminous efficiency function $V(\lambda)$ of the CIE standard photopic observer. The $V(\lambda)$ function was first adopted in 1924 by the CIE for $2^{\circ}$ viewing conditions, based on the work of Gibson and Tyndall. ${ }^{23}$ It corresponds to the spectral sensitivity of the long-wavelength-sensitive (L) and middle-wavelength-sensitive (M) cone photoreceptors and peaks at $555 \mathrm{~nm}$, solid gray line in Figure 1(a). It is applicable when light is plentiful, from 1 to $10^{6} \mathrm{~cd} \cdot \mathrm{m}^{-2}$. At lower light levels the rods become active and dominate from $10^{-6}$ to $10^{-2} \mathrm{~cd} \cdot \mathrm{m}^{-2}$, shifting the maximum spectral sensitivity towards shorter wavelengths. In between purely photopic and scotopic vision, at a luminance from $10^{-2}$ to $1 \mathrm{~cd} \cdot \mathrm{m}^{-2}$, both systems are active and this is called the mesopic condition. As the spectral sensitivity of rods and cones differ, the CIE adopted a standard scotopic luminous efficiency function $V^{\prime}(\lambda)$ for $20^{\circ}$ central visual field viewing conditions of the CIE standard scotopic observer in 1951, based on measurements by Wald ${ }^{24}$ and Crawford. ${ }^{25}$ The $V^{\prime}(\lambda)$ function is shown in Figure 1(a) denoted with a dotted gray line and has a peak wavelength around $507 \mathrm{~nm}$.

The CIE $1924 V(\lambda)$ function is still used today to derive photometric quantities, despite more recent improvements recommended by the CIE. ${ }^{26-28}$ Because of the known problems of the CIE $1924 V(\lambda)$ function, we use the $V(\lambda)$ appropriate for $10^{\circ}$ viewing condition, Figure 1(a), where its sensitivity is higher in the short-wavelength region as the macular pigment becomes more transparent with retinal eccentricity. The new $V_{10}(\lambda)$ function was obtained under neutral adaptation that corresponded to daylight adaptation (CIE standard illuminant D65) and is modeled as a weighted sum of the L- and M-cone fundamentals. ${ }^{29}$ The same L:M cone ratio of $2: 1$ is assumed to apply at $2^{\circ}$ and $10^{\circ}$ viewing conditions. ${ }^{28}$

A spectral weighting function for circadian non-visual responses, the circadian efficiency function $C(\lambda)$ was proposed by $\mathrm{Gall}^{30}$ using the effects on melatonin suppression as the indicator of spectral sensitivity based on measured data. ${ }^{1,2}$ This circadian efficiency function $C(\lambda)$ in Figure 1(a), has a peak sensitivity at $450 \mathrm{~nm}$ and was implemented in the German prestandard DIN V 5031-100:2009. ${ }^{31}$ Instead of being derived from either a single photoreceptor or a weighted combination thereof, this $C(\lambda)$ function was obtained from published graphs ${ }^{1,2}$ that had already been force-fit assuming a univariant action spectrum, hence potentially limiting the spectral interpretation of the data. As earlier studies support the hypothesis that the quantal sensitivity of melanopsin peaks near $480 \mathrm{~nm}$ in vitro, ${ }^{12}$ Enezi et al. ${ }^{13}$ proposed to adopt the melanopic spectral efficiency function $V^{z}(\lambda)$ in Figure 1(a). It was constructed using a mathematical template of a single opsin and peaks close to $490 \mathrm{~nm}$ when estimated in vivo for radiant flux. 
It has been shown that the ipRGCs have a dual role: 1) they receive and transmit signals from rods and cones, even in the absence of melanopsin; and 2) are independent photoreceptors via activation of melatonin, even in the absence of rods and cones. ${ }^{8,12,32-34}$ Therefore, instead of only evaluating the light stimulus to the ipRGCs, it is recommended to specify the light stimulus in terms of the five types of photoreceptors within the human retina. ${ }^{17}$ The rod photoreceptors contain a photopigment called rhodopsin with maximum absorbance wavelength $496.3 \mathrm{~nm}$. The cone photoreceptors contain different types of photopsin called erythrolabe, chlorolabe, and cyanolable for L-cones, M-cones, and S-cones with maximum absorbance wavelength $558.4 \mathrm{~nm}, 530.8 \mathrm{~nm}$, and $419 \mathrm{~nm}$, respectively. ${ }^{35}$ Figure 1(b) shows the spectral weighting functions of the five photoreceptors corrected for a 32 year old observer. Note that the correction due to lens transmittance and other ocular media causes the maximum peak to shift theoretically to longer wavelengths when estimated in vivo. In addition to shifts in spectral sensitivity, which become more apparent with age due to yellowing of the lens, it is also possible to account for the relative reduction in the total light amount received at the retina, for further explanation go to Section 3.4.

(a)

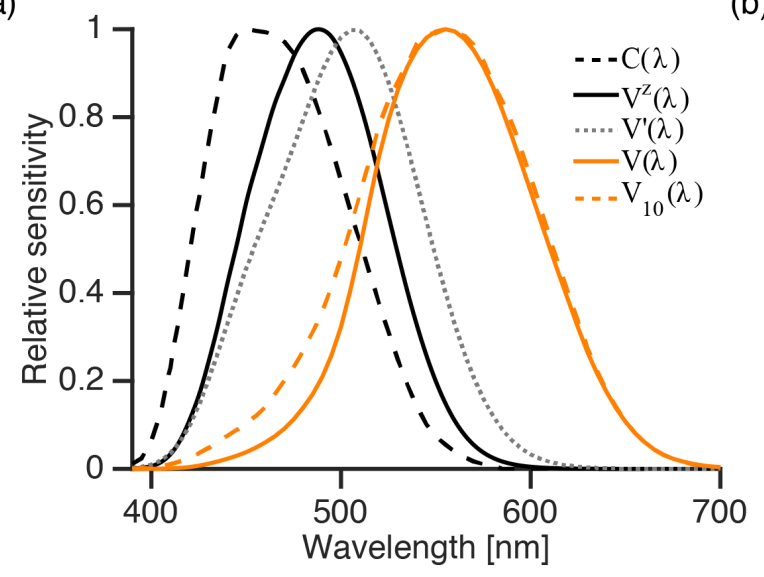

(b)

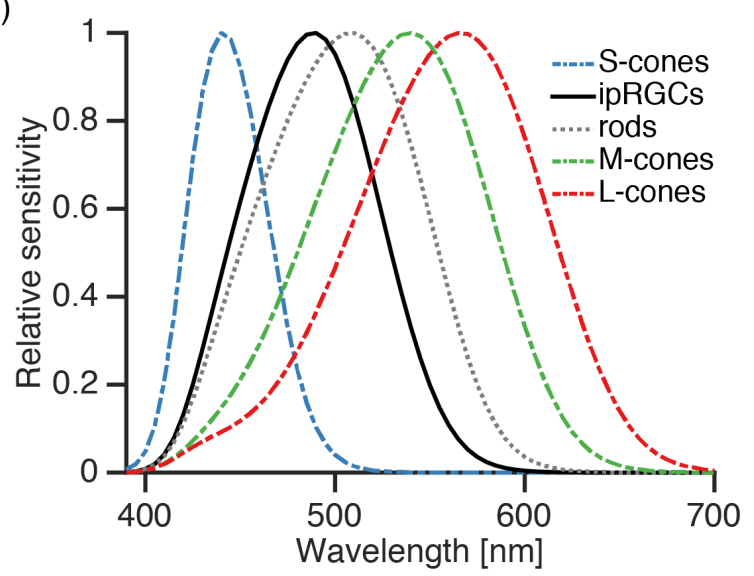

Figure 1 (a) The relative spectral sensitivity of the circadian $C(\lambda),{ }^{30}$ melanopic $V^{z}(\lambda),{ }^{13}$ scotopic $V^{\prime}(\lambda)$ (CIE 1951), photopic $V(\lambda)$ (CIE 1924), and for $10^{\circ}$ viewing condition photopic $V_{10}(\lambda)^{28}$ luminous efficiency functions. (b) The spectral sensitivity of the five human photoreceptors. The spectral sensitivity curves are constructed using an opsin template ${ }^{36}$ and a lens transmittance of a 32-yearold. ${ }^{37}$

Another model exists, the circadian light quantity (CL) developed by Rea et al., that combines the response of more than one photoreceptor depending on the spectral distribution of the light source, assuming that multiple photoreceptors work together as in brightness and/or color perception. ${ }^{38,39}$ The resulting spectral weighting function is neither smooth nor symmetric as the ones in Figure 1. 


\subsection{Towards non-visual quantities of light}

Since the discovery of the ipRGCs, new and revised quantities have been proposed to complement the existing radiometric and photometric measurement systems. ${ }^{13-19,38,40} \mathrm{We}$ have categorized these quantities row-wise depending on their application and column-wise based on whether the proposed quantity is derived in relation to the radiometric or the photometric system, see Table 1. As seen the information is scattered throughout the literature and it is not easy to understand the difference between these quantities because the terminology is not consistent. Although the categorization may seem trivial, it is crucial to provide users with a unified description to avoid confusion and misunderstanding across disciplines.

In the following sections, we will explain how to derive the quantities in Table 1 by category in more details and discuss the terminologies that have developed in parallel.

Table 1 Overview and categorization of existing quantities proposed for evaluating non-visual spectral effectiveness of light. The quantities are categorized row-wise based on their application and columnwise based the relation to the radiometric and the photometric systems.

\begin{tabular}{|c|c|c|}
\hline & Radiometric & Photometric \\
\hline \multirow[t]{3}{*}{ CIE system } & Irradiance & Photopic illuminance \\
\hline & Not possible & Circadian illuminance $^{14}$ \\
\hline & & Melanopic illuminance ${ }^{13}$ \\
\hline \multirow[t]{4}{*}{ Relative quantities } & Circadian efficiency $^{19,41}$ & $\begin{array}{l}\text { Relative ratio of circadian to } \\
\text { photopic lumens }\end{array}$ \\
\hline & & Circadian action factor ${ }^{15,19,30,31}$ \\
\hline & & Circadian potential ${ }^{16}$ \\
\hline & & Melatonin suppression index ${ }^{42}$ \\
\hline \multirow[t]{3}{*}{ Spectrally-weighted } & Circadian effective irradiance ${ }^{15,31}$ & $\alpha$-opic equivalent \\
\hline & Circadian light ${ }^{38,39}$ & illuminance $^{17,18}$ \\
\hline & a-opic irradiance ${ }^{18}$ & \\
\hline \multirow[t]{2}{*}{ Biologically-scaled } & None existing & Circadian equivalent \\
\hline & & illuminance $^{16}$ \\
\hline
\end{tabular}

\subsubsection{The CIE photometric system}

The conventional method for quantifying light is either in terms of radiant flux $\Phi_{\mathrm{e}}$ in units of power $[\mathrm{W}]$ or in irradiance $E_{\mathrm{e}}$ in units of power per area $\left[\mathrm{W} \cdot \mathrm{m}^{-2}\right]$. These quantities are purely physical and are called radiometric quantities. Photometric quantities are derived from the 
radiometric quantities based on the definition of luminous efficacy, calculated to simulate human visual responses to light, not the physical properties of light. The SI unit of luminous efficacy is lumens per watt $\left[\mathrm{lm} \cdot \mathrm{W}^{-1}\right]$. Photopic luminous efficacy $K(\lambda)$ has a maximum value of $K_{\mathrm{m}}=683 \mathrm{~lm} \cdot \mathrm{W}^{-1}$ for monochromatic light of wavelength $555 \mathrm{~nm}$ (green), where scotopic luminous efficacy $K^{\prime}(\lambda)$ reaches a maximum of $K_{\mathrm{m}}^{\prime}=1700 \mathrm{~lm} \cdot \mathrm{W}^{-1}$ for monochromatic light of wavelength $507 \mathrm{~nm}$, Figure 2. The luminous flux $\Phi_{\mathrm{v}}[\mathrm{lm}]$ falling on a unit area is called illuminance $E_{\mathrm{V}}$ and can be written as

$$
E_{\mathrm{v}}=K_{m} \int E_{\mathrm{e}, \lambda}(\lambda) V(\lambda) \mathrm{d} \lambda,
$$

measured in lux [lx]. For scotopic vision, the symbols in the equation above are replaced by $K_{\mathrm{m}}^{\prime}$ and $V^{\prime}(\lambda)$. Following these principles, the melanopic illuminance can be written as

$$
E_{\mathrm{v}}=K_{z, v} \int E_{\mathrm{e}, \lambda}(\lambda) V^{\mathrm{z}}(\lambda) \mathrm{d} \lambda,
$$

where $V^{2}(\lambda)$ is the melanopic spectral efficiency function (Figure 1(a)) and the melanopic luminous efficacy constant $K_{z, v}=4557 \mathrm{~lm} \cdot \mathrm{W}^{-1}$ ensures that, for illuminance at $555 \mathrm{~nm}$, melanopic illuminance is equal to photopic illuminance. ${ }^{13}$ While this method is the only way to be consistent with the conventional quantities used in photometry, it may not be practical for comparing responses of different photoreceptors types. Lang ${ }^{43}$ argues that this extremely high value, which is not related to a comparability of visual and non-visual effects in reality, would definitely lead to strong confusion in the application. This 'extremely high value' is due to the fact that the melanopic system $K^{2}(\lambda)$ has a much higher weight compared to the scotopic and photopic systems because the spectral luminous efficacy functions are normalized to $683 \mathrm{~lm} \cdot \mathrm{W}^{-1}$ at wavelength $555 \mathrm{~nm}$, see Figure 2.

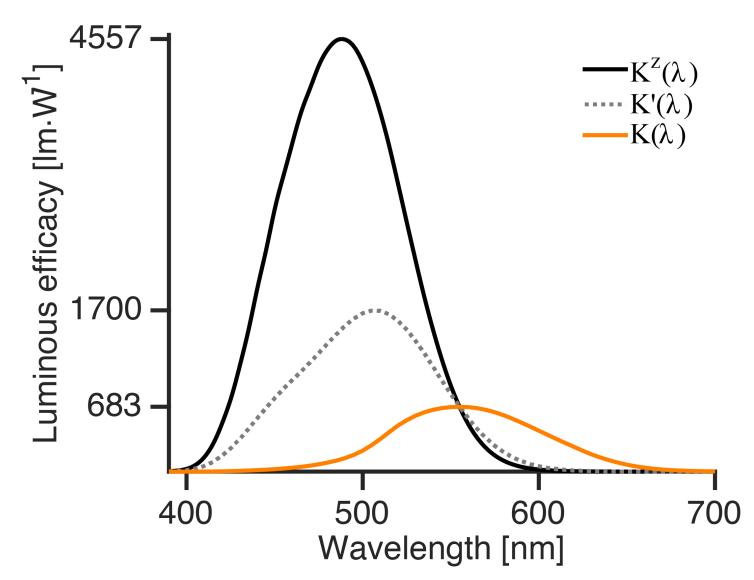

Figure 2 The luminous efficacy for melanopic $K^{z}(\lambda)$, scotopic $K^{\prime}(\lambda)$, and photopic $K(\lambda)$ vision. 


\subsubsection{Relative quantities}

Two methods of evaluating spectral effectiveness of light for a specific biological response are in absolute or relative terms. Absolute terms refer to physical quantities such as irradiance or illuminance reported in International System of Units (SI) and relative terms refer to unitless quantities obtained as ratios of absolute terms. Reporting the relative ratio between two different systems, i.e. the circadian and the photopic system, can be useful for comparing spectral power distributions (SPDs) of different light sources. ${ }^{15,16,40-42}$ These ratios provide estimates of the relative errors that would be generated if a conventional light measuring device employing the $V(\lambda)$ function were used to characterize input to the circadian system. ${ }^{40}$ Gall and Bieske ${ }^{15}$ introduced the circadian action factor to make it easy to transfer the absolute quantities to each other. The circadian action factor is defined as

$$
a_{\mathrm{c}, \mathrm{v}}=\frac{\int X_{\mathrm{e}, \lambda}(\lambda) C(\lambda) \mathrm{d} \lambda}{\int X_{\mathrm{e}, \lambda}(\lambda) V(\lambda) \mathrm{d} \lambda}
$$

where $X_{\mathrm{e}, \lambda}(\lambda)$ can be replaced with $\Phi_{\mathrm{e}, \lambda}(\lambda)$ or $E_{\mathrm{e}, \lambda}(\lambda)$ for calculating radiant flux or irradiance, respectively and $C(\lambda)$ is the circadian efficiency function, Figure 1(a). The $a_{\mathrm{c}, \mathrm{v}}$ factor together with the $C(\lambda)$ function have been used to compare the performance of different light sources. ${ }^{15,41,44}$

Others have developed a similar concept that looks at the circadian- or non-visual-weighted versus luminous-weighted irradiance, but using different procedures of normalization and/or generating a new spectral weighting function. For example the relative ratio of 'circadian' to photopic lumens ${ }^{40}$ and the melatonin suppression index (MSI). ${ }^{42}$ More importantly these quantities where all developed to evaluate spectral impacts of different SPDs in relation to illuminance. There also exists an example of where the circadian-weighted versus the total irradiance is proposed, called circadian efficiency by Bellia ${ }^{14}$ but it is not as widely used. The circadian efficiency of radiation is defined as

$$
\eta_{c}=\frac{\int x_{\mathrm{e}, \lambda}(\lambda) C(\lambda) \mathrm{d} \lambda}{\int x_{\mathrm{e}, \lambda}(\lambda) \mathrm{d} \lambda}
$$

in a recent CIE technical note, ${ }^{19}$ which summarizes definition of terms related to photobiological effects. It also includes the circadian factor of luminous radiation in equation (3).

\subsubsection{Spectrally-weighted quantities}

The spectrally-weighted quantities listed in Table 1 are calculated quantities used to compare against observed biological health effects to gain understanding of how the non-visual system 
reacts to different types of spectral light exposure. Ideally, these absolute quantities describe how effective a given light exposure is to produce an effect.

In 2009 the DIN pre-standard ${ }^{31}$ recommended a circadian effective value to be derived by weighting a radiometric quantity $X_{\mathrm{e}}(\lambda)$ with $C(\lambda)$

$$
X_{\mathrm{e}, \mathrm{c}}=\int X_{\mathrm{e}, \lambda}(\lambda) C(\lambda) \mathrm{d} \lambda,
$$

where $X_{\mathrm{e}, \lambda}(\lambda)$ can be replaced with $\Phi_{\mathrm{e}, \lambda}(\lambda)$ or $E_{\mathrm{e}, \lambda}(\lambda)$ for calculating radiant flux or irradiance, respectively. The subscript c on $X_{\mathrm{e}}$ represent a scaling with the $C(\lambda)$ curve and $\Phi_{\mathrm{e}, \mathrm{c}}$ or $E_{\mathrm{e}, \mathrm{c}}$ are given in equivalents of standard units $[\mathrm{W}]$ or $\left[\mathrm{W} \cdot \mathrm{m}^{-2}\right]$, respectively. The relation to photometric values $X_{\mathrm{v}}$ is obtained as

$$
X_{\mathrm{e}, \mathrm{c}}=K_{\mathrm{m}}^{-1} \times X_{\mathrm{v}} \times a_{\mathrm{c}, \mathrm{v}} .
$$

The issue with this method is that if the $C(\lambda)$ function is replaced with a new sensitivity function that may better approximate circadian non-visual responses, the $a_{\mathrm{c}, \mathrm{v}}$ values may change significantly. ${ }^{41}$ Therefore it is difficult to understand the performance of the biological response under consideration and especially when comparing different spectral sensitivity functions.

Apart from calculating the melanopic illuminance, equation (2), or the circadian effective irradiance, equation (5), another solution has been suggested that defines a new absolute measure. ${ }^{17}$ Instead of normalizing the spectral efficiency functions so that the maximum height is equal to one, it was proposed to normalize the sensitivity curves to the area of the $V(\lambda)$ function. This is done separately for each type of photoreceptor using the sub notation $\alpha$ to distinguish between the different types, $\alpha$ can take the value $z, l c, m c, s c$ and $r$, respectively for melanopic, erythropic, chloropic, cyanopic, and rhodopic equivalent illuminance. In addition, five new units have to be introduced so that one equivalent $\alpha$-opic illuminance is equal to one photopic illuminance under equal-energy light conditions. The new equivalent $\alpha-$ opic illuminance is obtained as

$$
E_{\alpha}=K_{\mathrm{N}} \int E_{\mathrm{e}, \lambda}(\lambda) N_{\alpha}(\lambda) \mathrm{d} \lambda,
$$

where $N_{\alpha}(\lambda)$ is the spectral sensitivity curve for photoreceptor $\alpha$ normalized to unity-peak and $K_{\mathrm{N}}=72983.25 \alpha-\mathrm{lm} \cdot \mathrm{W}^{-1}$ is a normalization constant derived by integrating the $V(\lambda)$ function multiplied by the photopic luminous efficacy or $K_{\mathrm{N}}=K_{\mathrm{m}} \int V(\lambda) \mathrm{d} \lambda=\int K(\lambda) \mathrm{d} \lambda$. Note that the value of $K_{\mathrm{N}}$ does not change with photoreceptor type $\alpha$ but the unit of the quantity changes accordingly. Consequently five new units are introduced: melanopic $[z-1 \mathrm{x}]$, erythropic $[l c-1 \mathrm{x}]$, chloropic $[m c-1 \mathrm{x}]$, cyanopic $[s c-1 \mathrm{x}]$ and rhodopic $[r-1 \mathrm{x}]$ illuminances; which is not consistent with the technical terminology already in use. ${ }^{20}$ 
More recently, the CIE TN 003:2015 ${ }^{18}$ recommended that the $\alpha$-opic irradiance should be used instead of the $\alpha$-opic equivalent illuminance to be consistent with the present definition of photobiological quantities. The $\alpha$-opic irradiance is determined by convolving the spectral irradiance, $E_{e, \lambda}(\lambda)\left[\mathrm{W} \cdot \mathrm{m}^{-2} \cdot \mathrm{nm}^{-1}\right]$, for each wavelength, with the action spectrum normalized to one at its peak. The mathematical formulation is the same as in equation (5) but the symbols differ by the photopigment labels. Note that the DIN pre-standard has been replaced according to the recent CIE recommendations. ${ }^{45}$

It is useful to provide a selection between energy- and vision-related spectrally-weighted quantities as provided, but not applying the same method for normalizing the spectral functions between these two approaches is conflicting. ${ }^{18}$ Whereas it is argued that the spectral weighting functions must be kept dimensionless, the issue of unequal weighting of photoreceptors is unsolved. The equal-area normalization does not generate a physical quantity and can be interpreted as having a dimensionless 'numerical' excitation effect that is the same under different light stimuli. The normalized sensitivity curves represent how much light 'hits' different types of photoreceptors with equal probability when exposed to pure white light. This approach does not describe the functional relationship by which light induces its effect, since stimulating each photoreceptor equally will not necessarily result in equal biological impact. ${ }^{17}$ The equal-area type of normalization is widely used in color science. Boyton and Kambe suggested a 'luminance normalization', for example, which applies when the S-cone spectral sensitivity is set so that one luminance unit of an equal-energy white light source produces one S-cone excitation unit. ${ }^{46}$

\subsubsection{Biologically-scaled (predicted) quantities}

Biologically-scaled quantities are calculated quantities to ensure that different types of spectral light exposure predict equivalent health effects. They are based on a calculation procedure that aims to predict how much light is needed to ensure the same outcome, instead of quantifying how effective the light stimuli is in producing an effect (i.e. the spectrallyweighted quantities). When replacing an old light source, it can be useful to convert illuminance values from a reference (old) light source to a target (new) light source while maintaining an equivalent stimulus to a specific photoreceptor. This concept, called the equivalent illuminance in the present paper, was first published by Pechacek et al. ${ }^{16}$ for one type of reference light source and with respect to one type of spectral sensitivity curve. The equivalent illuminance value was then calculated using the subjective alertness intensityresponse curve from Cajochen et $a l^{47}$ and the calculated circadian potential value. The 
circadian potential $\mathrm{W}-C(\lambda)$ was derived by first scaling the SPDs of the light source to equal illuminance and then weighting it with the $C(\lambda)$ function. ${ }^{16}$ This method of scaling light quantities was described for a specific design case and demonstrated numerically. In Section 3.3 it is demonstrated how this concept can be generalized into mathematical formulation within the proposed framework.

The biologically-scaled quantities are practical for assessment and regulation of light exposure because they are directly measureable in standard SI units. The limitation of this approach is that it is based on the current knowledge, which is observed biological effects under certain lighting conditions. The results are then generalized to real world settings based on fixed assumptions about the underlying sensitivity function that have yet to be validated.

\section{Unified framework}

The unified framework was developed to explain the relationship between existing quantities and their application to research and in practice. In order to provide a complete description of non-visual spectral effectiveness of light in both radiometric and photometric related terms, we developed a new unit-less factor, the relative spectral effectiveness (RSE) factor. The new factor enables the evaluation of a SPD of a light source in terms of its comparative 'brightness' or 'energy' relationship to an equal-energy spectrum for any system of photoreception.

As spectral weighting functions are necessary for evaluating visual and non-visual responses to light, we start by describing how we derived the spectral weighting functions, Section 3.1. The mathematical concepts of the new factor and the relation between absolute and relative quantities are explained in Sections 3.2 and 3.3, respectively. As the standard observer is often assumed to be in the age of 32 years old, a factor to correct for reduced retinal exposure due to age is introduced in Section 3.4. Section 3.5 summarizes the added value and general structure of the unified framework.

\subsection{Selection and normalization of spectral weighting functions}

The spectral sensitivity curves for the five human photoreceptors: ipRGCs, L-cones, M-cones, S-cones, and rods are shown in Figure 3(a). These curves are constructed from the maximum absorbance wavelength $\lambda_{\max }$ using an opsin template ${ }^{36}$ and a lens transmittance model of a 32 year old observer ${ }^{37}$ corresponding to the standard human observer. The influence of the macula is not considered, since it mainly protects cones in the fovea. The axial optical densities of peripheral cones and rods are set to 0.2 and 0.4 , respectively. ${ }^{48,49}$ The cone value 
is lower compared to optical density of central cones because optical density declines with eccentricity. The axial optical density value for the ipRGCs is not known and therefore not considered here. Note that these curves are used to demonstrate the use of the unified framework and should be replaced or adjusted when more information is available. The method is described in more details in the appendix.

The spectral weighting functions or action spectra are commonly normalized to a value of one at the wavelength of 'maximum actions' but we recommend normalizing the area under the curve to one, Figure 3(a). The same approach was adopted to scale the five human photoreceptors by Lucas et al. ${ }^{17}$ to calculate the equivalent rhodopic, erythropic, chloropic, cyanopic and melanopic illuminance values, see Section 2.2.3. The sensitivity curves in Figure 3(a) are shown for wavelengths in the visible range from $390 \mathrm{~nm}$ to $700 \mathrm{~nm}$. Although the human eye is sensitive to light $<390 \mathrm{~nm}$ and $>700 \mathrm{~nm}$, the relative sensitivity at these wavelengths is extremely low $<0.005$ when normalized to equal-area.

(a)

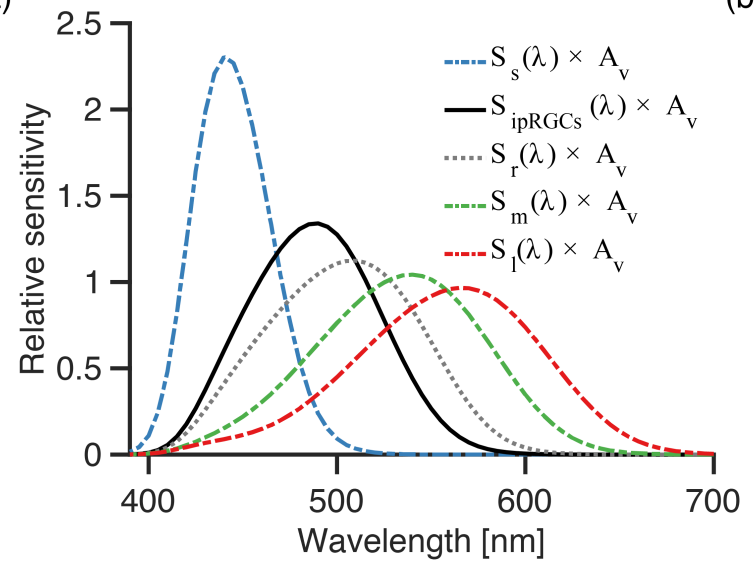

(b)

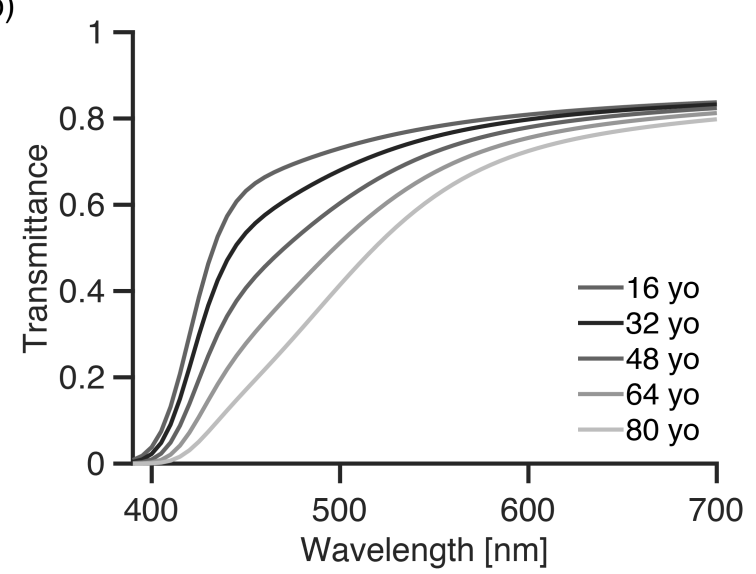

Figure 3 (a) Spectral weighting functions of the five photoreceptors scaled to have equal area under the curve. (b) Spectral distribution of the total transmittance of the aging human eye computed via the model of van de Kraats and van Norren. ${ }^{37}$ The lens gradually yellows and darkens with age: the lens of an 80 year old filters out approximately twice as much of short wavelength light as the lens of a 16 year old.

\subsection{Formulation of the relative spectral effectiveness factor}

The unit-less factor is called the relative spectral effectiveness (RSE) factor with subscripts $\mathrm{V}$ and e that represent photopic vision and energy relations, respectively. The vision-related $\mathrm{RSE}_{\mathrm{v}, \mathrm{i}}$ factor determines the relationship between the weighted spectral irradiance with a spectral sensitivity function $S_{\mathrm{i}}(\lambda)$ and the spectral irradiance weighted with the $V_{10}(\lambda)$ function. 
The vision-related $\mathrm{RSE}_{\mathrm{v}, \mathrm{i}}$ factor for any $S_{\mathrm{i}}(\lambda)$ weighted spectral irradiance is obtained as follows

$$
\operatorname{RSE}_{\mathrm{v}, \mathrm{i}}=\frac{\int_{\lambda_{1}}^{\lambda_{2}} E_{\mathrm{e}, \lambda}(\lambda) S_{\mathrm{i}}(\lambda) \mathrm{d} \lambda}{\int_{\lambda_{1}}^{\lambda_{2}} E_{\mathrm{e}, \lambda}(\lambda) V_{10}(\lambda) \mathrm{d} \lambda} \times A_{\mathrm{v}},
$$

where $A_{\mathrm{v}}$ is a normalization constant equal to the area of $\int_{\lambda_{1}}^{\lambda_{2}} V_{10}(\lambda) d \lambda$. The letter $i$ is a general notation and can for example take five forms: ipRGC, $l, m, s$ and $r$ that stands for ipRGCs, L-cones, M-cones, S-cones or rods, respectively. The respective sensitivity functions are noted as $S_{\text {ipRGC }}(\lambda), S_{1}(\lambda), S_{\mathrm{m}}(\lambda), S_{\mathrm{s}}(\lambda)$, and $S_{\mathrm{r}}(\lambda)$, shown in Figure 3(a).

An exclusive focus on how spectral sensitivity is rated in relation to the photopic visual system may not be the best practice. Instead of evaluating the non-visual responses as a subsystem or as an extension of the photometric system, they can be evaluated directly in relation to the radiometric system independently of the $V_{10}(\lambda)$ function. The spectral irradiance can simply be weighted with a spectral sensitivity curve. More specifically, the energy-related $\mathrm{RSE}_{\mathrm{e}, \mathrm{i}}$ factor is the relationship between the weighted spectral irradiance $S_{\mathrm{i}}(\lambda)$ and the nonweighted spectral irradiance

$$
\mathrm{RSE}_{\mathrm{e}, \mathrm{i}}=\frac{\int_{\lambda_{1}}^{\lambda_{2}} E_{\mathrm{e}, \lambda}(\lambda) S_{\mathrm{i}}(\lambda) \mathrm{d} \lambda}{\int_{\lambda_{1}}^{\lambda_{2}} E_{\mathrm{e}, \lambda}(\lambda) \mathrm{d} \lambda} \times A_{\mathrm{e}},
$$

where $A_{\mathrm{e}}$ is a normalization constant equal to $\lambda_{2}-\lambda_{1}$, derived by integrating the area of an rectangle with height equal to one on the interval from $\lambda_{1} \mathrm{~nm}$ to $\lambda_{2} \mathrm{~nm}$. Since $S_{\mathrm{i}}(\lambda)$ integrates to one in the wavelength range, the area normalization constants, $A_{\mathrm{v}}$ and $A_{\mathrm{e}}$, ensure equations (8) and (9) to return unity if the spectral power distribution of the light source has an equalenergy at each wavelength.

\subsection{Relation between absolute and relative quantities}

The RSE factor can be used to both calculate spectrally-weighted and biologically-scaled quantities. The spectrally-weighted effective irradiance $\left[\mathrm{W} \cdot \mathrm{m}^{-2}\right.$ (effective)] is obtained by multiplying the bounded irradiance by the energy-related RSE factor for photoreceptor $\mathrm{i}$

$$
E_{\mathrm{e}, \mathrm{i}}^{\mathrm{eff}}=E_{\mathrm{e}, \lambda_{1}-\lambda_{2}} \times \mathrm{RSE}_{\mathrm{e}, \mathrm{i}} \text {. }
$$

In a similar way, the spectrally-weighted effective illuminance [1x (effective)] is obtained by multiplying the photopic illuminance by the vision-related RSE factor

$$
E_{\mathrm{v}, \mathrm{i}}^{\mathrm{eff}}=E_{\mathrm{v}, \lambda_{1}-\lambda_{2}} \times \mathrm{RSE}_{\mathrm{v}, \mathrm{i}}
$$

The biologically-scaled equivalent irradiance $\left[\mathrm{W} \cdot \mathrm{m}^{-2}\right]$ is defined as 


$$
E_{\mathrm{e}, \mathrm{i}}^{\mathrm{eq}}=E_{\mathrm{e}, \lambda_{1}-\lambda_{2}} \times r_{\mathrm{e}, \mathrm{i}}
$$

where $r_{\mathrm{e}, \mathrm{i}}$ is the ratio of the energy-related RSE factor for the reference light source, $\mathrm{RSE}_{\mathrm{e}, \mathrm{i}}^{\mathrm{ref}}$, to the target light source, $\mathrm{RSE}_{\mathrm{e}, \mathrm{i}}^{\mathrm{tar}}$,

$$
r_{\mathrm{e}, \mathrm{i}}=\frac{\mathrm{RSE}_{\mathrm{e}, \mathrm{i}}^{\mathrm{ref}}}{\operatorname{RSE}_{\mathrm{e}, \mathrm{i}}^{\mathrm{tar}}}
$$

The $E_{e, \lambda_{1}-\lambda_{2}}$ corresponds to the irradiance of the reference light source that induces the desired biological effect in the wavelength range $\lambda_{1}$ and $\lambda_{2}$. The $E_{e, i}^{e q}$ is the equivalent irradiance for the target light source to stimulate a certain photoreceptor equally as the reference light source did before. The biologically-scaled equivalent irradiance is a predicted quantity based on assumptions regarding the non-visual system. These assumptions must be verified through experimental studies but remain useful for extrapolating current findings. The equivalent illuminance by Pechacek et al. ${ }^{16}$ as described in Section 2.2.4 can be written in a mathematical form based on the definition of the vision-related RSE and extended to handle different types of reference light sources and photoreceptors. The biologically-scaled equivalent illuminance $[1 \mathrm{x}]$ is defined as

$$
E_{\mathrm{v}, \mathrm{i}}^{\mathrm{eq}}=E_{\mathrm{v}, \lambda_{1}-\lambda_{2}} \times r_{\mathrm{v}, \mathrm{i}}
$$

where $r_{\mathrm{v}, \mathrm{i}}$ is the ratio of the vision-related RSE factor for the reference light source $\mathrm{RSE}_{\mathrm{V}, \mathrm{i}}^{\mathrm{ref}}$ to the target light source RSE $\mathrm{v}_{\mathrm{v}, \mathrm{i}}^{\mathrm{tar}}$

$$
r_{\mathrm{v}, \mathrm{i}}=\frac{\mathrm{RSE}_{\mathrm{v}, \mathrm{i}}^{\mathrm{ref}}}{\operatorname{RSE}_{\mathrm{v}, \mathrm{i}}^{\mathrm{tar}}}
$$

The $E_{v, \lambda_{1}-\lambda_{2}}$ corresponds to the illuminance of the reference light source that induces the desired biological effect in the wavelength range $\lambda_{1}$ and $\lambda_{2}$. The $E_{v, i}^{e q}$ is equivalent illuminance for the target light source to stimulate photoreceptor equally as the reference light source did before.

The equivalent illuminance is similar to the concept of 'equivalent luminance' that was introduced by the CIE as a supplement to the photometric system to scale brightness under mesopic lighting conditions. ${ }^{50}$ According to the CIE definition, "the equivalent luminance is the luminance of a specified reference light that has the same brightness as the target light under consideration." Building upon this argument, the meaning of the word 'equivalent' is used differently by Lucas et al. ${ }^{17}$ and Pechacek et al. ${ }^{16}$ The concept of the equivalent $\alpha$-opic illuminances used by Lucas et al. ${ }^{17}$ is similar to the circadian effective irradiance. ${ }^{15,31}$ From here on effective illuminance will be used instead of equivalent $\alpha$-opic illuminance to avoid 
confusion. Instead of considering this vision-related brightness response, we instead consider non-visual biological responses which is reasonable because the underlying non-visual spectral sensitivity is based on a combination of multiple photoreceptors ${ }^{4}$ although how they interact in relation to intensity, duration, timing, and light history is not yet fully understood. While many non-visual responses exist, the challenge is that some are more easily observed than others, and most studies have not been designed or analyzed with a multi-photoreceptor system in mind.

The spectrally-weighted effective irradiance is recommended for evaluating the spectral effectiveness of light sources rather than the effective illuminance, to avoid the issue of not being consistent with the definition of luminous efficacy. The advantage of using biologically-scaled equivalent quantities over spectrally-weighted effective quantities is that it provides a quantity in conventional SI units, for example irradiance $\left[\mathrm{W} \cdot \mathrm{m}^{-2}\right]$ and photopic illuminance [lx]. These quantities can be translated directly to adjust lighting in experimental or architectural settings using a lux meter. The equivalent $\alpha$-opic illuminance or effective illuminance are quantities that cannot be directly applied without a new device that can measure spectrally-weighted illuminance. Moreover, as $\alpha-1 \mathrm{x}$ is subject to a sensitivity curve, it means that a new unit is defined for every new sensitivity curve. This property is not typical for the SI unit system, as many quantities are often measured in the same unit. To name the units by the procedure used to derive the quantities, rather than the photoreceptor type, is more practical. In this paper, we will use [spectrally-weighted $\left.\mathrm{W} \cdot \mathrm{m}^{-2}\right]$ or $\left[\mathrm{W} \cdot \mathrm{m}^{-2}(\right.$ effective $\left.)\right]$ and [spectrally-weighted $1 \mathrm{x}]$ or [ $\mathrm{lx}$ (effective)] for all spectrally-weighted quantities, which is consistent with optical radiation exposure metrics used in photobiology. ${ }^{20}$ The names of spectral weighting function should be used in the quantity name, so spectrally-weighted quantities for ipRGCs are called ipRGC effective irradiance $\left[\mathrm{W} \cdot \mathrm{m}^{-2}\right.$ (eff.)] and ipRGC effective illuminance [lx (eff.)].

\subsection{Age correction factor}

It is important to note that all structures in the eye can absorb, reflect, and scatter light depending on their optical characteristics. The lens becomes less transparent with age as illustrated in Figure 3(b). A way to estimate the age-related loss in light amount due to lens transmittance is to estimate the percentage reduction separately for each light source. ${ }^{31}$ The age correction factor $\mathrm{k}_{\mathrm{i}}$ for a light source and spectral weighting function $S_{i}(\lambda)$ can be approximated for the observer's age A as 


$$
k_{i}(A)=\frac{\int_{\lambda_{1}}^{\lambda_{2}} E_{e, \lambda} \frac{\tau(\lambda, A)}{\tau(\lambda, A=32)} S_{i}(\lambda) d \lambda}{\int_{\lambda_{1}}^{\lambda_{2}} E_{e, \lambda} S_{i}(\lambda) d \lambda},
$$

where $\tau$ is the transmittance of optical eye media, see equation (19) in Appendix. ${ }^{37,51}$ For younger age than 32 years old the correction factor will be higher than one and for older age the correction factor will be lower, as less light gets to the retina. The influence of the change in lens transmittance with aging on loss in light amount is obtained by the multiplication of the age correction factor and the spectrally-weighted effective quantities.

\subsection{Integrated perspective}

The RSE factor was introduced as a unit-less factor, which shows the relative relationship between spectrally-weighted light quantities and the standard CIE radiometric and photometric quantities. The mathematical description of the new factor is designed to return unity if the spectral power distribution of a light source has an equal energy spectrum.

Applying the unified framework to a light source with a non-equal energy spectrum will result in a low value $(<1)$ of the RSE factor if the light source has a lower stimulus potential than the equal energy light source for the photoreceptive system under consideration. If the light source has a higher stimulus potential than the equal energy light source, the RSE factor will be larger than one $(>1)$.

The application of the RSE factor is illustrated in Figure 4 for a conceptual understanding of the various ways of evaluating spectral effectiveness of ocular light exposure. The focus of this paper is on the spectral sensitivity of the five human photoreceptors, where the framework could be expanded to other photobiological systems. When selecting a light source, it is useful to evaluate how its spectral profile may stimulate the photoreceptor types differently. The equal-area normalization of the spectral weighting functions allows us to compare the sensitivities of any photoreceptive systems and avoids the problem related to the unity-peak normalization where resulting values are highly influenced by the total area of the curves, which is unequal, and cannot be directly compared for effectiveness. For the evaluation of spectral effectiveness for human health we are most concerned with energy content from $390 \mathrm{~nm}$ to $700 \mathrm{~nm}$.

In order to compare spectral profiles of light sources in an absolute quantity instead of a relative scale, the RSE factor can be used in two ways. On one hand to calculate spectrallyweighted quantities from CIE radiometric and photometric quantities given the RSE factor of a light source specific to a photoreceptor type. On the other hand, the RSE factor can be turned into a set of conversion ratios that determine irradiance or illuminance levels resulting 
in equivalent stimulus to a specific photoreceptive system for any light sources. This is useful for converting illuminance levels from one particular reference light source to equivalent illuminance levels in another target light source. The RSE factor thus enables the evaluation of the relative spectral power distribution of a light source in terms of its comparative 'brightness' relationship to an equal-energy spectrum for any system of photoreception. An age correction factor (Section 3.4) was added to predict the relative loss in light amount received at the retina.

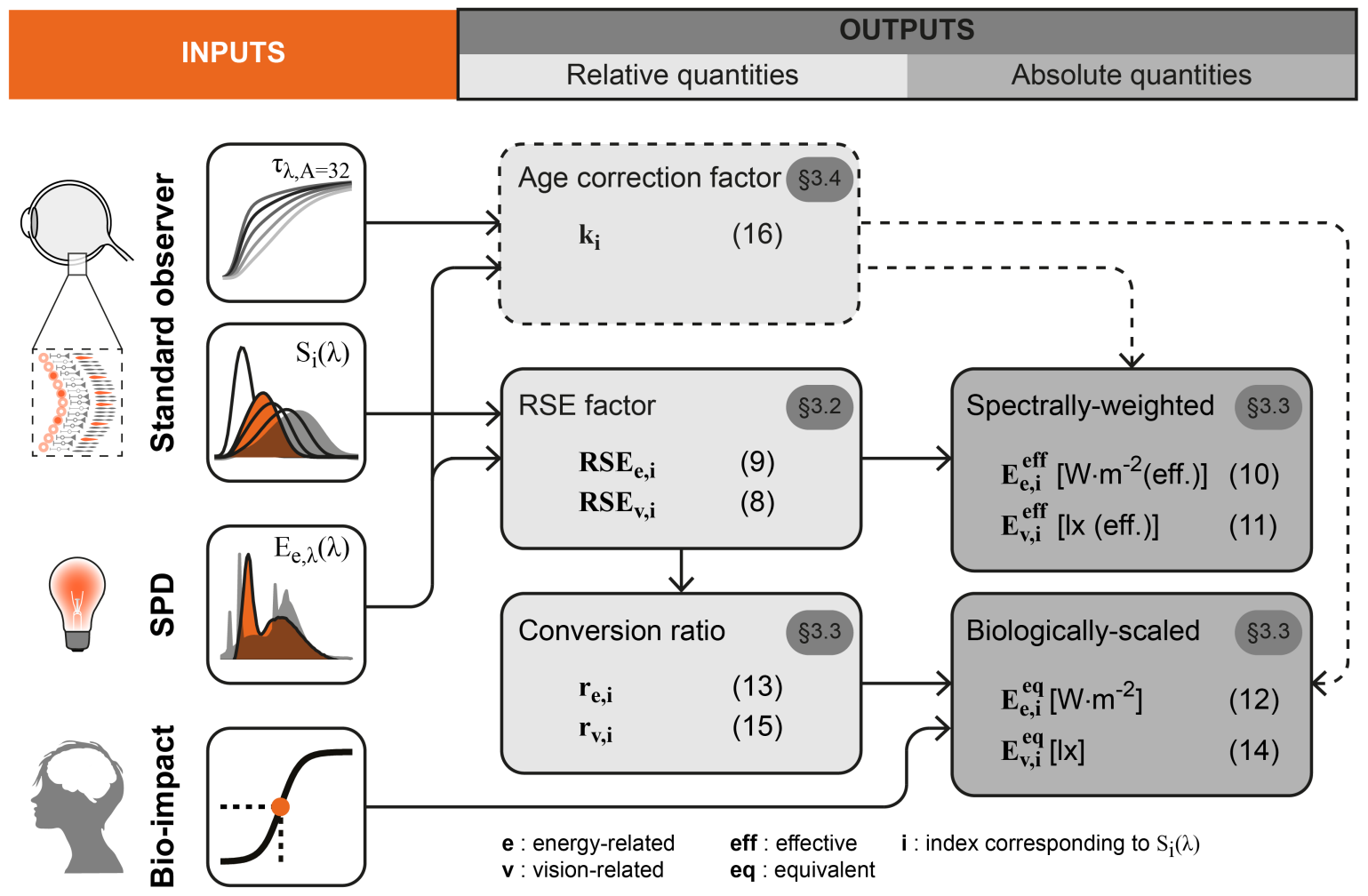

Figure 4 Schematic of the unified framework. The framework outputs both relative and absolute quantities. Equal-area normalization of spectral weighting functions is applied to define a new unit-less factor, called the relative spectral effectiveness (RSE) factor. The RSE factor shows the relative relationship between spectrally-weighted quantities and radiometric or photometric quantities. It can be used to compare the non-visual spectral effectiveness of different light sources for different photoreceptors and to derive biologically-scaled quantities based on known experimental results.

A common trend when a new quantity is introduced is to add a prefix to the following unit. For example for spectrally weighted quantities new units have appeared in literature such as cir-lm, ${ }^{40} \mathrm{~b}-1 \mathrm{~m},{ }^{43}$ and $\mathrm{z}-1 \mathrm{x} .{ }^{17}$ When giving a quantitative amount, it is essential to specify which quantity is meant and which spectral weighting function was used, as the unit should not change. The unit of spectrally-weighted quantities used here is specified [W. $\mathrm{m}^{-2}$ (effective)] or $\left[\mathrm{W} \cdot \mathrm{m}^{-2}\right.$ (eff.)] for irradiance related quantities. 
In this paper, we only report irradiance and illuminance values, but the same principle applies to other radiometric or photometric quantities, respectively. The spectrally-weighted effective quantities can be directly calculated using the pre-calculated RSE factor for any given irradiance or illuminance. So, when tuning of light exposure intensity needs to be monitored, it is not necessary to measure the SPD if it can be assumed that the spectral profile does not change. It should also be noted that irrespective of the arguments against illuminance measurements, a large part of practitioners could relate light to illuminance better than to any other value. Equivalent illuminance is more likely to be recognized by the investigator than effective illuminance.

\section{Case studies using SpeKtro}

In order to demonstrate the utility of the proposed framework, we will discuss four hypothetical cases that span a range of interests for researchers, designers and developers in lighting and related fields. The unified framework has been implemented in SpeKtro. The SpeKtro dashboard is a new tool publicly available on-line at spektro.epfl.ch to import and analyze different SPD according to the unified framework. The following sections demonstrate the use of relative and absolute terms. Relative quantities are more commonly used in applied physics and optics ${ }^{42,44}$ to compare electric light sources (Section 4.1) and absolute quantities are usually reported in biological studies (Section 4.2). ${ }^{13,17}$ The uses of the two are often combined in real-world applications of lighting research and design (Section $4.3-4) .{ }^{14-16}$

It is necessary to know the SPD of a light source to determine its relative spectral effectiveness accurately. In the following examples we use six electric light sources: incandescent $2856 \mathrm{~K}$ (CIE A), three-band fluorescent $4000 \mathrm{~K}$ (CIE F11), white LED $6500 \mathrm{~K}$ (LED65), equal-energy $5454 \mathrm{~K}$ (CIE E), broadband fluorescent $6500 \mathrm{~K}$ (CIE F7), and blue LED $9500 \mathrm{~K}$ (LED95). These light sources where selected to illustrate how the RSE factor changes in relation to different types of light sources. Their SPDs are shown in Figures 5(a) and 5(b) for the visible part of the electromagnetic spectrum, between $390 \mathrm{~nm}$ and $700 \mathrm{~nm}$, scaled to deliver equal illuminance of $330 \mathrm{~lx}$. By constraining the wavelength range to $390 \mathrm{~nm}$ to $700 \mathrm{~nm}$, the effectiveness factor is fully focused on the spectral effectiveness over the visible range. It is assumed that irradiance outside $390 \mathrm{~nm}$ to $700 \mathrm{~nm}$ does not contribute to biological or non-visual effects of ocular light exposure in humans, which is subject to change. 
(a)

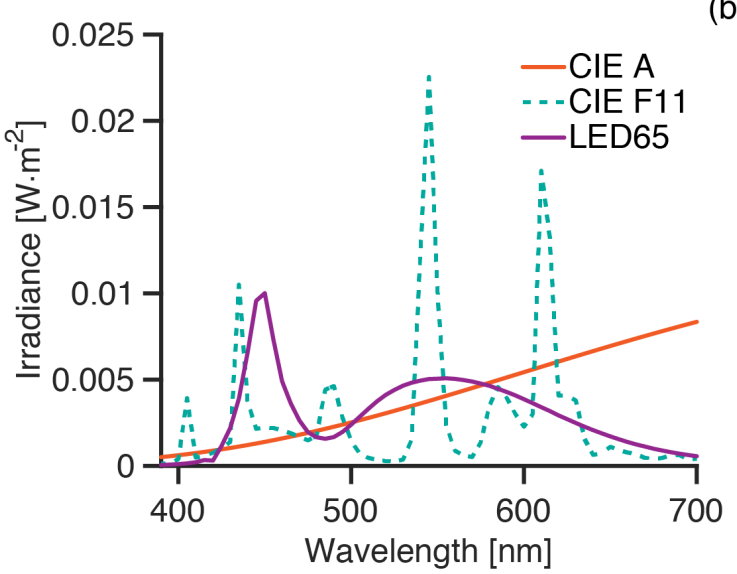

(b)

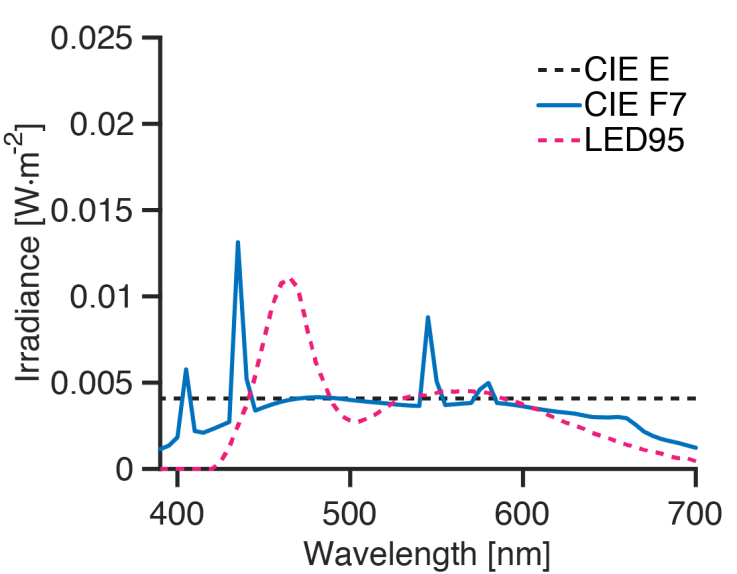

Figure 5 (a) The relative SPDs for incandescent $2856 \mathrm{~K}$ (CIE A), three-band fluorescent $4000 \mathrm{~K}$ (CIE F11), white LED $6500 \mathrm{~K}$ (LED65), (b) equal-energy $5454 \mathrm{~K}$ (CIE E), broadband fluorescent $6500 \mathrm{~K}$ (CIE F7), and blue LED $9500 \mathrm{~K}$ (LED95). The SPDs are tuned to illuminance of $330 \mathrm{~lx}$, their respective irradiances are $1.24 \mathrm{~W} \cdot \mathrm{m}^{-2}, 0.89 \mathrm{~W} \cdot \mathrm{m}^{-2}, 0.94 \mathrm{~W} \cdot \mathrm{m}^{-2}, 1.26 \mathrm{~W} \cdot \mathrm{m}^{-2}, 1.11 \mathrm{Wm} \mathrm{m}^{-2}$, and $1.04 \mathrm{~W} \cdot \mathrm{m}^{-2}$ within the wavelength range from $390 \mathrm{~nm}$ to $700 \mathrm{~nm}$.

\subsection{Comparing electric light sources}

In this section, the six electric light sources are compared for the five human photoreceptors, first, using two different methods of normalizing the spectral weighing functions and then two different methods of scaling the relative effectiveness. Figure 6(a) and 6(b) show the relative relations between the photoreceptors by applying the unity-peak normalization. This method is referred here as the circadian action factor ${ }^{15,30}$ and was first used to compare the circadian weighted spectrum to the photopic weighted spectrum. In comparison, Figure 6(c) and 6(d) show the proposed vision-related $\mathrm{RSE}_{v, i}$ factor values for the five photoreceptors. Values above one indicate that the corresponding light sources are more effective than an equalenergy spectrum (CIE E) at stimulating the photoreceptors at the same illuminance. Light sources that are rich in the short-wavelength part of the spectrum are shown to be more effective at stimulating the ipRGC than other light sources. In this example, the CIE F7 and LED95 light sources are more effective than the CIE A, CIE F11, and LED65 light sources. For example the incandescent (CIE A) light source returns a value of 0.54 for ipRGCs, which means that with such a source twice the illuminance is necessary to give the same ipRGC effectiveness as the fluorescent (CIE F7) light source that has a factor value close to 1 . The ranking of light sources obtained using the vision-related $\mathrm{RSE}_{\mathrm{v}, \mathrm{ipRGC}}$ factor values compared to the circadian action factor shows the same relative relations within photoreceptor types. The comparison between photoreceptor types is distorted, however. Figure 6(a) and 
6(b) show the ratio of the spectrally-weighted irradiance to luminous-weighted irradiance when the weighting functions are normalized to unity peak, which is in line with the principle behind the 'circadian action factor'. The action factor for equal-energy (CIE E) light source shows the relationship between the curve shapes of the spectral weighting functions, while the vision-related $\mathrm{RSE}_{\mathrm{v}, \mathrm{i}}$ factor returns equal measure for any type of sensitivity curve for CIE $\mathrm{E}$. The S-cone action factor values in Figure 6(a) and 6(b) are lower than the action factors for the other photoreceptors because the S-cone spectral weighting function is the narrowest. Therefore, the resulting RSE factor values are more easily understood when using the equalarea normalization.

Instead of evaluating light sources in relation to the photopic visual system, it is possible to omit the $V(\lambda)$ function and calculate the relative spectral effectiveness of irradiance. In Figure 6(e) and 6(f) the energy-related RSE factors are shown for ipRGCs, L-M-S-cones, and rods. The energy-related RSE factors are very different compared to the vision-related RSE factors. The results are no longer smoothed by the $V(\lambda)$ function and the influence of the different spectral profiles is more visible. This is seen when Figures 6(c) and 6(e) are compared side by side, the distribution of the energy-related RSE factor values for the different photoreceptor types spans a wider range than the vision-related RSE factor values. Of the illuminants listed, the CIE F7 illuminant is the most homogenous stimulus (after CIE E), as it has the least spread in effectiveness. 
(a)

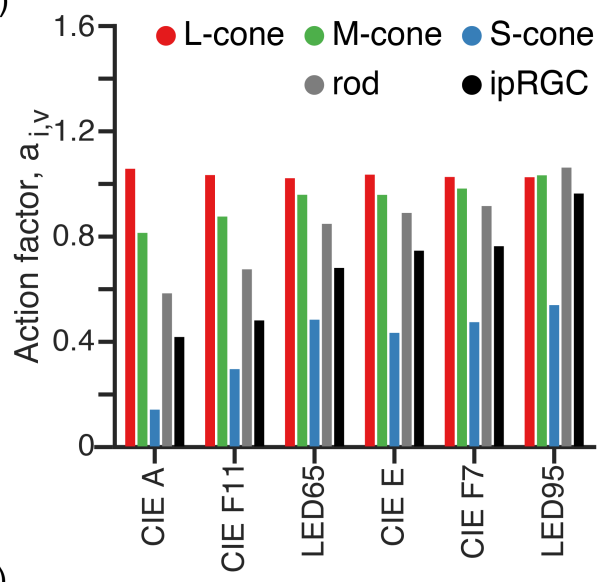

(c)

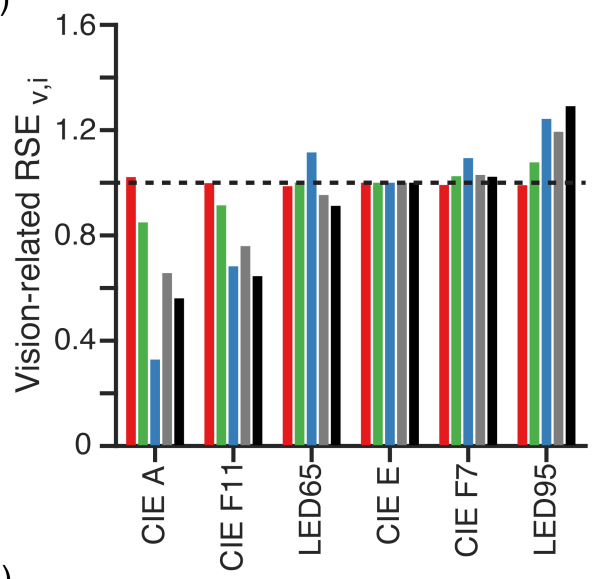

(e)

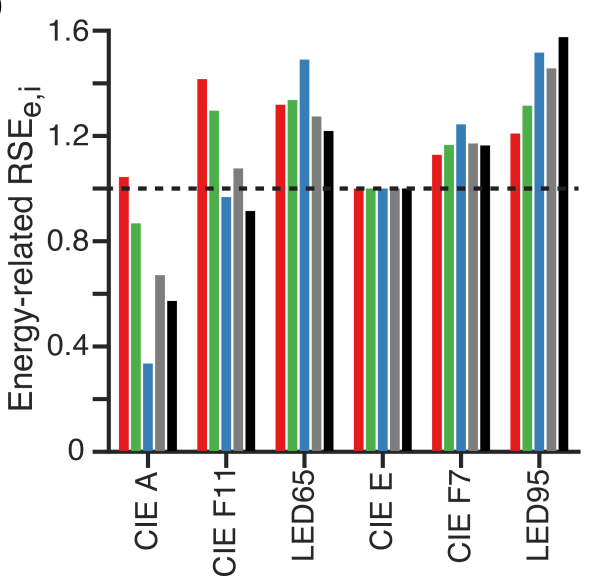

(b)

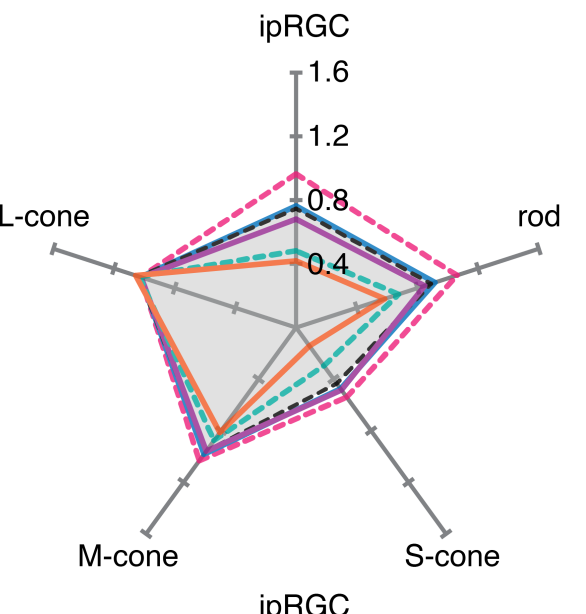

$\nabla \operatorname{CIEA}$

i- CIEF11

$\bigcirc$ LED65

Q⿱宀八 CIEE

$\checkmark$ CIEF7

b) LED95

(d)

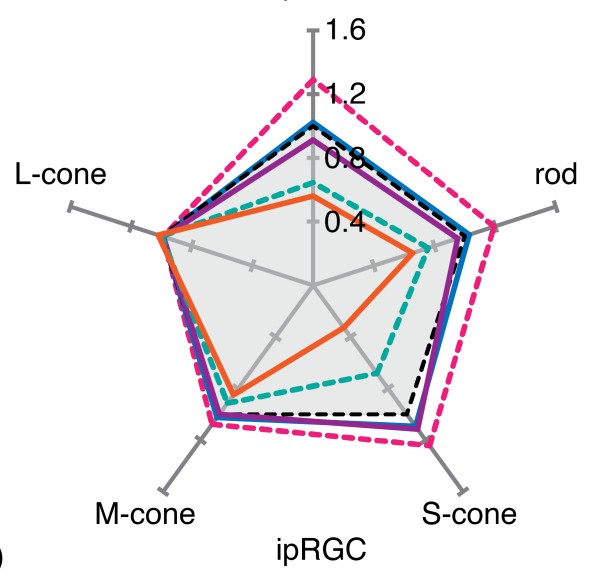

$\nabla$ CIE A

Lj CIE F11

$\square$ LED65

i.j CIE E

$\triangle$ CIE F7

i. LED95

(f)

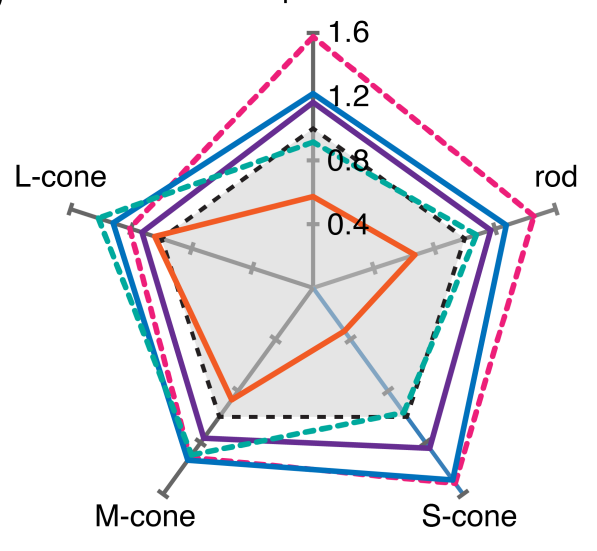

- CIE A

i CIE F11

$\square$ LED65

$\because$ CIEE

$\bigcirc$ CIE F7
? LED95

Figure 6 The bar plots show the results for (a) action factor (unity-peak normalization), (c) visionrelated RSE factor and (e) energy-related RSE factor grouped per light source. The same data is visualized in the radar plots (b), (d) and (f), respectively.

The LED65 and CIE F7 light sources in Figure 6 have the same correlated color temperature

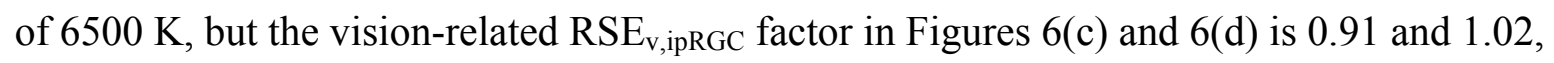
respectively, and the energy-related $\mathrm{RSE}_{\mathrm{e}, \mathrm{ipRC}}$ factor in Figure 6(e) and 6(f) is 1.22 and 1.16. This difference illustrates a well-known problem in the use of color temperature alone in the specification of a light source. 


\subsection{Evaluating non-visual responses}

Knowledge of photoreceptor spectral sensitivities is important for the understanding and modeling of non-visual responses to light and practical applications of lighting design and light measurements for experimental research. Defining the relative roles and contributions of the different photoreceptors types in non-visual responses to light remains a challenge. ${ }^{17}$ The magnitude of the non-visual effects of light depends on the spectral power distribution of the light source and on the dynamic changes in light exposure, which are not yet fully understood. ${ }^{52}$ In order to begin the process, it is assumed that the sensitivity of the photoreceptors does not change with lighting conditions, so each photoreceptor can be evaluated separately. As more is learned about the dynamics of photoreceptor recruitment with different light conditions (intensity, wavelength, duration, timing, and light history), the relative contributions of the photoreceptors can be scaled and summed as appropriate.

Certain non-visual responses may be composed of one or more spectral weighting functions. The RSE factor can be used to explore how ranking of light sources changes by combining and weighing the signals of different photoreceptor types. For simplicity, we assume that the spectral weighting functions of the five human photoreceptors do not change with different states of adaptation, unless the photopigment is bleached at high intensity levels. The contribution of each photoreceptor to non-visual responses varies, however, with adaption to different light exposures. As pointed out the $V(\lambda)$ function is only valid under a limited range of conditions, as it reflects the sum of the L- and M-cone sensitivities. The ultimate goal is to predict the magnitude of non-visual response by quantifying the light stimulus.

Given that the non-visual responses are dependent on more than one type of photoreceptor, we can compare how their overall sensitivity sums up to predict the effectiveness of light sources. For example Gooley et al. ${ }^{4}$ suggest that at the beginning of a light exposure, cones and ipRGCs contribute approximately equally to non-visual responses. By assuming that the spectral sensitivity of cones is described by $V_{10}(\lambda)$, we can stack the results of the RSE factor, Figure 7(a), to visualize the combination of the two RSE factor values with equal weights, Figure 7(b). If the total spectral irradiance is not equal between the light sources, then it is necessary to compare the spectrally-weighted effective irradiance. Figure 7(c) shows the spectrally-weighted effective irradiance values, given the total spectral irradiance values in Figure 5 and the RSE factor values in Figure 7(a). As seen, the luminous effective irradiance is equal for all light sources, because they are tuned to equal illuminance. If we apply equal weights to the results in Figure 7(c) the ranking of the light sources in Figure 7(d) changes 
compared to the relative values in Figure 7(b), in particular that the LED65 and F11 shift down by two steps.

(a)

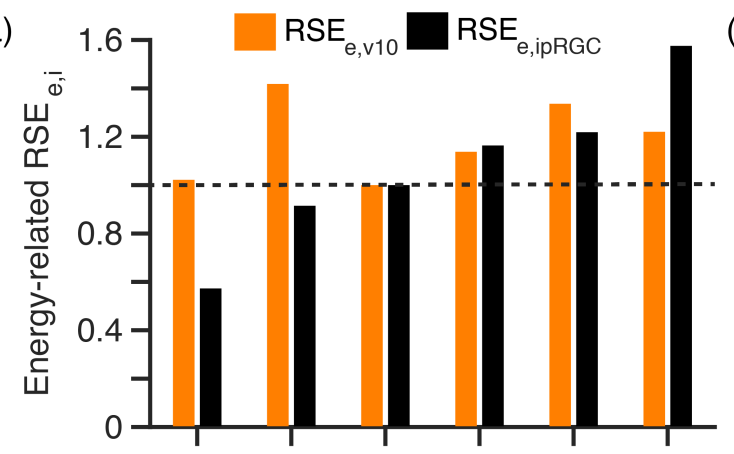

(c)

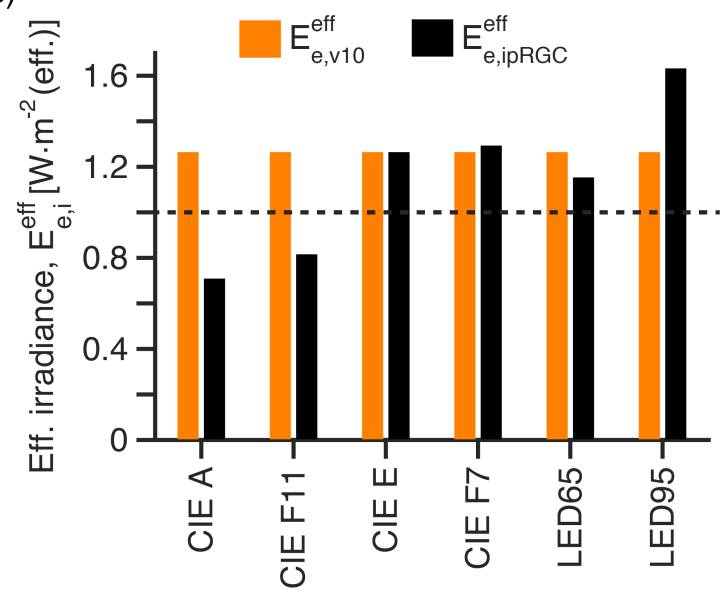

(b)

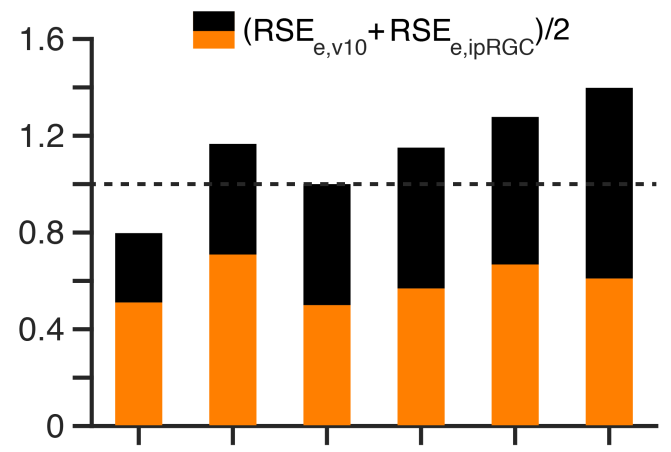

- $\left(\mathrm{E}_{\mathrm{e}, \mathrm{v} 10}^{\mathrm{eff}}+\mathrm{E}_{\mathrm{e}, \mathrm{ipRGC}}^{\mathrm{eff}}\right) / 2$

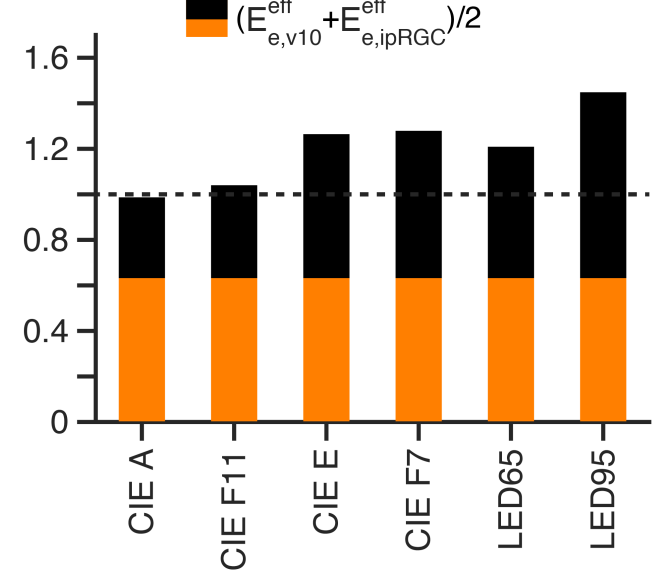

Figure 7 (a) The energy-related RSE factor for $V_{10}(\lambda)$ and ipRGC. (b) The combined and weighted energy-related RSE factor for $V_{10}(\lambda)$ and ipRGC from Figure $7(a)$, each assigned with weight equal to 0.5. (c) The $V_{10}(\lambda)$ and ipRGC effective irradiance corresponding to the total irradiance values given in Figure 5. (d) The combined and weighted effective irradiance from Figure 7(c).

\subsection{Predicting intensity thresholds}

The main advantages of using the RSE factor is that once a factor value is calculated, energyrelated or vision-related absolute quantities can be calculated for a given irradiance or illuminance. The spectrally-weighted quantities may appear unfamiliar and need perhaps more time to integrate into practice than research. For this purpose, we will demonstrate how the conversion ratios are useful to predict intensity levels for different types of light sources. The calculation of biologically-scaled equivalent illuminances is based on a known intensityresponse relationship. Figure 8(a) shows ipRGC equivalent illuminance values for the reference light source CIE F11. These values are the equivalent illuminance of light exhibiting a non-visual response that corresponds to a selected percentage of the desired effect achieved by the reference light source. The illuminance levels of the reference light 
source (CIE F11) are selected to be 95 lx, 128 1x, and 328 lx (dotted lines) that correspond to $50 \%, 75 \%$, and $99 \%$ derived from the subjective alertness intensity-response relationship in Figure $8(\mathrm{~b})$, published by Cajochen et al ${ }^{47}$ This procedure can be adapted to any intensityresponse curve, given that the SPD of the light source is known. The results presented here should not be taken as guidelines for design, as the goal is to demonstrate the use of the framework.

The target equivalent illuminance can easily be compared to visual design criteria, ${ }^{53}$ as the equivalent illuminance is reported in the conventional unit lux. The change in equivalent illuminance from 328 lx of CIE F11 that achieved near maximum effective intensity (99\%) is illustrated in Figure 8(a). For example 207 lx of CIE F7 would induce the same ipRGC effectiveness as 328 1x of CIE F11 although the same visual stimulation would not be obtained.

(a)

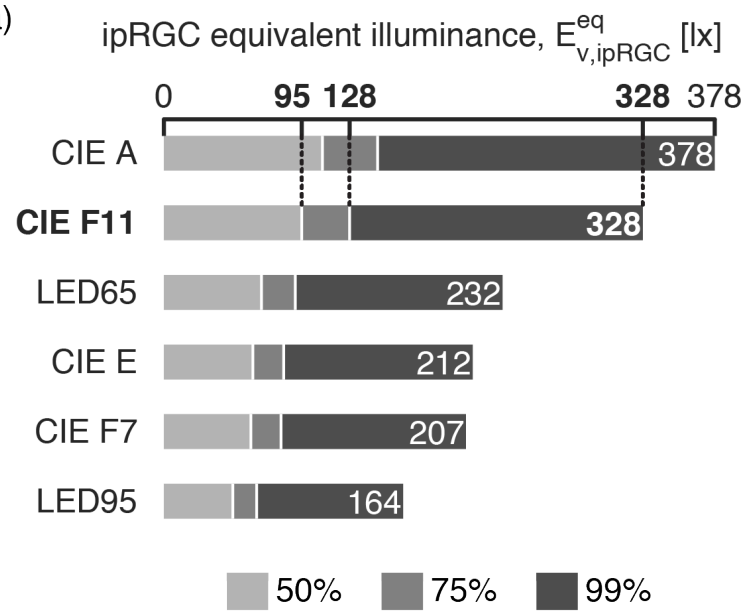

(b) Subjective alertness intensity-response

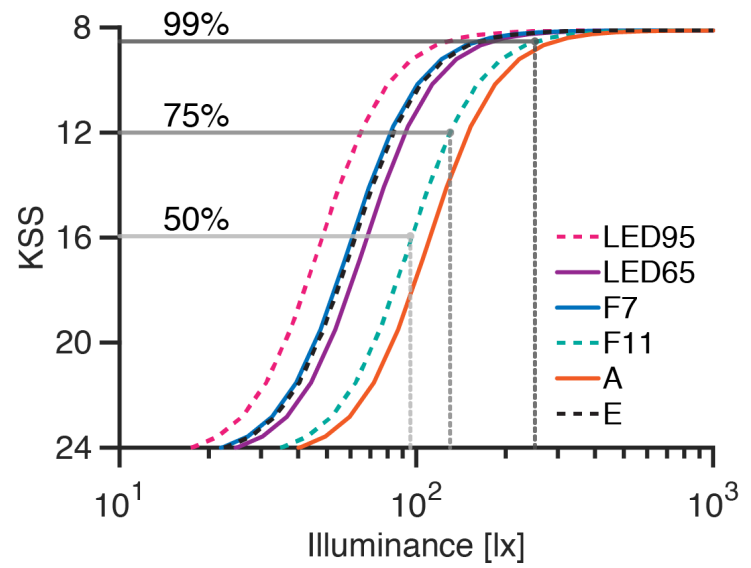

Figure 8 (a) The ipRGC equivalent illuminance for CIE F11 illuminant of $95 \mathrm{Ix}, 128 \mathrm{Ix}$, and $330 \mathrm{Ix}$, represented with light to dark colours, respectively, corresponding to the intensity-response curve in (b). KSS stands for Karolinska Sleepiness Scale.

\subsection{Predicting the relative loss in retinal exposure due to age}

The results presented in Sections 4.1 to 4.3 are calculated for the standard human observer $(\mathrm{A}=32)$. As discussed in Section 3.1 the lens filters the light that reaches the retina and does not only influence the spectral distribution but also the total amount of light that reaches the retina, see Section 3.4. The shape of the relative spectral sensitivity does not change much as a function of age, where there is a small shift to longer wavelengths. Therefore, the relative spectral effectiveness does not change significantly for the selected light sources but because 
the age-dependent effect is more visible for monochromatic light sources, it must be more carefully evaluated.

For each light source we estimated an age correction factor for 16, 48, 64, and 80 years old observers. Table 2 shows the relative change for ipRGCs effective irradiance of the different light sources. The combined effect of reduced lens transmittance predicts ca. $50 \%$ reduction in effective irradiance between 32 and 80 years of age. The reduction is more apparent for light sources rich in the blue part of the spectrum. These results are based on the theoretical assumption that as the aging lens decreases the total amount of light received at the retina. However, there is evidence that the effect of aging on spectral sensitivity to light of the circadian system cannot be fully explained by reduction in lens transmittance. Najjar et al. ${ }^{54}$ suggest that during healthy aging a compensatory mechanism takes place to maintain an optimal non-visual sensitivity to light.

Table 2 The age correction factor for ipRGCs effective irradiance of illuminants for different age groups.

\begin{tabular}{crrrrrr} 
Age (A) & CIE A & CIE F11 & CIE E & CIE F7 & LED65 & LED95 \\
\hline $\mathbf{1 6}$ & 1.09 & 1.11 & 1.11 & 1.11 & 1.12 & 1.12 \\
$\mathbf{3 2}$ & 1.00 & 1.00 & 1.00 & 1.00 & 1.00 & 1.00 \\
$\mathbf{4 8}$ & 0.87 & 0.85 & 0.85 & 0.85 & 0.84 & 0.84 \\
$\mathbf{6 4}$ & 0.73 & 0.69 & 0.68 & 0.68 & 0.67 & 0.66 \\
$\mathbf{8 0}$ & 0.59 & 0.55 & 0.53 & 0.52 & 0.51 & 0.50
\end{tabular}

The age factor values in Table 2 take into account optical properties of the eye that may influence the spectral sensitivity in relative terms but we do not consider the total amount of light loss. It has been estimated that less than $50 \%$ of the light entering the human eye reaches the retina and less than $10 \%$ is absorbed in the retina. ${ }^{55}$ Moreover, the amount of light that enters the eye is regulated by the pupil, a non-visual response to light in itself. This means that the average diameter of the pupil adapts to the effective light stimuli, causing reduction in retinal exposure without influencing the spectral transmittance. The problem is that the 'effective' light has not been defined with an action spectrum.

\section{Conclusion}

This paper describes a unified framework to explore non-visual spectrum lighting for human health. It was developed to eliminate redundancy and explain in a structured way, useful for various scientific communities, how to evaluate and report the spectral effectiveness of 
different photoreceptors or photoreceptive systems using a revised terminology. The unified framework introduces a unit-less factor, relative spectral effectiveness (RSE) factor, based on the concept of equal-area normalization given that the functional role of each group of photoreceptor type is not yet understood. By giving them equal perceptual weight in the nonvisual system, it is argued to be more consistent than the 'traditional' way of normalizing to maximum peak. In particular an improved set of quantities and naming conventions permits the calculation of non-visual effective irradiance using relative weights to account for inputs from multiple photoreceptors.

As demonstrated in the paper, the equal-area approach allows for a clearer connection between the spectral specification and the underlying physiology than the unity-peak approach, which remains the most common representation of spectral sensitivities and is useful for comparing relative differences in peak sensitivity, not the magnitude of a response. This unifying effort builds on existing literature and aims to help practitioners and researchers to interpret and communicate information on non-visual spectral effectiveness in a universal way.

\section{Funding}

This work was carried out with the support from École Polytechnique Fédérale de Lausanne (EPFL) and from the Swiss National Science Foundation (SNSF), project-153018. SWL was supported in part by the National Space Biomedical Research Institute through NASA NCC $9-58$.

\section{Acknowledgement}

The authors wish to thank Thomas Denoréaz for programming the website spektro.epfl.ch and Lorenzo Cantelli for the technical support.

\section{References}

1. Brainard GC, Hanifin JP, Greeson JM, et al. Action spectrum for melatonin regulation in humans: Evidence for a novel circadian photoreceptor. J Neurosci 2001; 21: 6405-6412.

2. Thapan K, Arendt J, Skene DJ. An action spectrum for melatonin suppression: evidence for a novel non-rod, non-cone photoreceptor system in humans. $J$ Physiol 2001; 535: 261267.

3. Chellappa SL, Steiner R, Blattner P, et al. Non-Visual Effects of Light on Melatonin, Alertness and Cognitive Performance: Can Blue-Enriched Light Keep Us Alert? PLoS ONE 2011; 6: e16429. 
4. Gooley JJ, Rajaratnam SMW, Brainard GC, et al. Spectral responses of the human circadian system depend on the irradiance and duration of exposure to light. Sci Transl Med 2010; 2: 31ra33.

5. Revell VL, Arendt J, Terman M, et al. Short-Wavelength Sensitivity of the Human Circadian System to Phase-Advancing Light. J Biol Rhythms 2005; 20: 270-272.

6. Cajochen C, Münch M, Kobialka S, et al. High sensitivity of human melatonin, alertness, thermoregulation, and heart rate to short wavelength light. J Clin Endocrinol Metab 2005; 90: 1311-1316.

7. Lockley SW, Evans EE, Scheer FA, et al. Short-wavelength sensitivity for the direct effects of light on alertness, vigilance, and the waking electroencephalogram in humans. Sleep 2006; 29: 161-168.

8. Vandewalle G, Gais S, Schabus M, et al. Wavelength-dependent modulation of brain responses to a working memory task by daytime light exposure. Cereb Cortex 2007; 17 : 2788-2795.

9. Gamlin PD, McDougal DH, Pokorny J, et al. Human and macaque pupil responses driven by melanopsin-containing retinal ganglion cells. Vision Res 2007; 47: 946-954.

10. Gooley JJ, Ho Mien I, St. Hilaire MA, et al. Melanopsin and rod-cone photoreceptors play different roles in mediating pupillary light responses during exposure to continuous light in humans. J Neurosci Off J Soc Neurosci 2012; 32: 14242-14253.

11. Provencio I, Rodriguez IR, Jiang G, et al. A novel human opsin in the inner retina. $J$ Neurosci 2000; 20: 600-605.

12. Berson DM, Dunn FA, Takao M. Phototransduction by Retinal Ganglion Cells That Set the Circadian Clock. Science 2002; 295: 1070-1073.

13. Enezi J al, Revell V, Brown T, et al. A 'melanopic' spectral efficiency function predicts the sensitivity of melanopsin photoreceptors to polychromatic lights. J Biol Rhythms 2011; 26: 314-323.

14. Bellia L, Bisegna F. From radiometry to circadian photometry: A theoretical approach. Build Environ 2013; 62: 63-68.

15. Gall D, Bieske K. Definition and measurement of circadian radiometric quantities. In: proceedings of the CIE Symposium 2004 on Light and Health: Non-Visual Effects. Vienna, Austria: Technical University of Ilmenau, 2004, pp. 129-132.

16. Pechacek CS, Andersen M, Lockley SW. Preliminary method for prospective analysis of the circadian efficacy of (day)light with applications to healthcare architecture. LEUKOS 2008; 5: 1-26.

17. Lucas RJ, Peirson SN, Berson DM, et al. Measuring and using light in the melanopsin age. Trends Neurosci 2014; 37: 1-9.

18. CIE. Report on the First International Workshop on Circadian and Neurophysiological Photometry, 2013. Technical Note CIE TN 003:2015. 
19. CIE. Relating Photochemical and Photobiological Quantities to Photometric Quantities. Technical Note CIE TN 002:2014.

20. Sliney DH. Radiometric Quantities and Units Used in Photobiology and Photochemistry: Recommendations of the Commission Internationale de l'Eclairage (International Commission on Illumination). Photochem Photobiol 2007; 83: 425-32.

21. Amundadottir ML, Lockley SW, Andersen M. A unified framework for evaluating nonvisual spectral effectiveness of ocular light exposure: key concepts. In: Proceedings of the 28th Session of the CIE. Manchester, UK: Commission Internationale de L'Eclairage, 2015, pp. 121-130.

22. CIE. CIE S 017/E:2011 ILV: International Lighting Vocabulary. http://eilv.cie.co.at/term/17 (2011, accessed 10 September 2015).

23. Gibson KS, Tyndall EPT. Visibility of radiant energy. Sci Papers Bur Stand 1923; 19 : 131-191.

24. Wald G. Human Vision and the Spectrum. Science 1945; 101: 653-658.

25. Crawford BH. The Scotopic Visibility Function. Proc Phys Soc Sect B 1949; 62: 321.

26. Judd DB. Report of US secretariat committee on colorimetry and artificial daylight. In: Proceedings of the Twelfth Session of the CIE. Stockholm, Sweden: Bureau Central de la CIE Paris, 1951, p. 11.

27. Vos JJ. Colorimetric and photometric properties of a 2-deg fundamental observer. Color Res Appl 1978; 3: 125-128.

28. Sharpe LT, Stockman A, Jagla W, et al. A luminous efficiency function, V( $\lambda)$, for daylight adaptation. $J$ Vis 2005; 5: 3.

29. CIE. Fundamental Chromaticity Diagram with Physiological Axes - Part 1. Technical Report CIE 170-1:2006.

30. Gall D. Circadiane Lichtgrössen und deren messtechnische Ermittlung. Licht 2002; 54 : 1292-1297.

31. DIN. DIN V 5031-100:2009-06 Optical radiation physics and illuminating engineering Part 100: Non-visual effects of ocular light on human beings - Quantities, symbols and action spectra. DIN V 5031-100:2009-06, Berlin: Beuth Verlag GmbH, June 2009.

32. Czeisler CA, Shanahan TL, Klerman EB, et al. Suppression of Melatonin Secretion in Some Blind Patients by Exposure to Bright Light. N Engl J Med 1995; 332: 6-11.

33. Hattar S, Lucas RJ, Mrosovsky N, et al. Melanopsin and rod-cone photoreceptive systems account for all major accessory visual functions in mice. Nature 2003; 424: 76-81.

34. Güler AD, Altimus CM, Ecker JL, et al. Multiple photoreceptors contribute to nonimageforming visual functions predominantly through melanopsin-containing retinal ganglion cells. Cold Spring Harb Symp Quant Biol 2007; 72: 509-515. 
35. Dartnall H. The interpretation of spectral sensitivity curves. Br Med Bull 1953; 9: 24-30.

36. Govardovskii VI, Fyhrquist N, Reuter T, et al. In search of the visual pigment template. Vis Neurosci 2000; 17: 509-528.

37. van de Kraats J, van Norren D. Optical density of the aging human ocular media in the visible and the UV. J Opt Soc Am A 2007; 24: 1842.

38. Rea MS, Figueiro MG, Bullough JD, et al. A model of phototransduction by the human circadian system. Brain Res Rev 2005; 50: 213-228.

39. Rea M, Figueiro M, Bierman A, et al. Circadian light. J Circadian Rhythms 2010; 8: 110.

40. Rea MS, Figueiro MG, Bullough JD. Circadian photobiology: An emerging framework for lighting practice and research. Light Res Technol 2002; 34: 177-187.

41. Bellia L, Bisegna F, Spada G. Lighting in indoor environments: Visual and non-visual effects of light sources with different spectral power distributions. Build Environ 2011; 46: 1984-1992.

42. Aubé M, Roby J, Kocifaj M. Evaluating potential spectral impacts of various artificial lights on melatonin suppression, photosynthesis, and star visibility. PLoS ONE 2013; 8: e67798.

43. Lang D. Energy efficient lighting for the biological clock. In: Proceedings of SPIE. San Francisco, California. Epub ahead of print 2011. DOI: 10.1117/12.875323.

44. Kozakov R, Franke S, Schöpp H. Approach to an effective biological spectrum of a light source. LEUKOS 2008; 4: 255-263.

45. DIN. DIN SPEC 5031-100 Optical radiation physics and illuminating engineering - Part 100: Non-visual effects of ocular light on human beings - Quantities, symbols and action spectra. DIN SPEC 5031-100:2015-08, Berlin: Beuth Verlag GmbH, August 2015.

46. Boynton RM, Kambe N. Chromatic Difference Steps of Moderate Size Measured along Theoretically Critical Axes. Color Res Appl 1980; 5: 13-23.

47. Cajochen C, Zeitzer JM, Czeisler CA, et al. Dose-response relationship for light intensity and ocular and electroencephalographic correlates of human alertness. Behav Brain Res 2000; 115: 75-83.

48. Sharpe LT, Stockman A, Knau H, et al. Macular pigment densities derived from central and peripheral spectral sensitivity differences. Vision Res 1998; 38: 3233-3239.

49. Lamb TD. Photoreceptor spectral sensitivities: Common shape in the long-wavelength region. Vision Res 1995; 35: 3083-3091.

50. Sagawa K. Toward a CIE supplementary system of photometry: brightness at any level including mesopic vision. Ophthalmic Physiol Opt 2006; 26: 240-245. 
51. CIE. A Computerized Approach to Transmission and Absorption Characteristics of the Human Eye (incl. Erratum 1). Technical Report CIE 203:2012 incl. Erratum 1.

52. Amundadottir ML, St. Hilaire MA, Lockley SW, et al. Modeling non-visual responses to light: unifying spectral sensitivity and temporal characteristics in a single model structure. In: CIE Centenary Conference 'Towards a New Century of Light'. Paris, France:

Commission Internationale de L'Eclairage, 2013, pp. 101-110.

53. Andersen M, Mardaljevic J, Lockley SW. A framework for predicting the non-visual effects of daylight - Part I: photobiology- based model. Light Res Technol 2012; 44: 3753.

54. Najjar RP, Chiquet C, Teikari P, et al. Aging of Non-Visual Spectral Sensitivity to Light in Humans: Compensatory Mechanisms? PLoS ONE 2014; 9: e85837.

55. Baylor DA. Photoreceptor signals and vision. Proctor lecture. Invest Ophthalmol Vis Sci 1987; 28: 34-49.

56. Alpern M. Lack of uniformity in colour matching. J Physiol 1979; 288: 85-105.

\section{Appendix}

\section{Construction of spectral weighting functions}

The spectral weighting functions used in the paper were constructed by adapting a standard procedure that was originally developed to approximate cone spectral sensitivities of any individual observer for different viewing conditions. The values for $S_{\mathrm{i}}(\lambda)$ used in this paper can be exported from the web site spektro.epfl.ch.

The procedure used in the paper for constructing a spectral sensitivity curve from the maximum absorbance wavelength $\lambda_{\max }$ to corneal spectral sensitivity is as follows:

\section{Low density absorbance}

The sensitivity of a single photopigment depends on the light wavelength being absorbed and its absorption spectrum can be approximated with a common template using only one parameter, the maximum absorbance wavelength $\lambda_{\max }{ }^{35}$

The template proposed by Govardovskii et al. ${ }^{36}$ was applied to construct the low density absorbance spectrum in quantal units for the photopigment. The template is expressed as

$$
S_{\mathrm{q}, \mathrm{D}}(x)=1 /\left(e^{a(A-x)}+e^{b(B-x)}+e^{c(C-x)}+D\right)+A_{\beta} \cdot e^{-\left(\frac{\lambda-\lambda_{\beta}}{d}\right)^{2}},
$$


where $\mathrm{x}=\lambda_{\max } / \lambda$, with the parameters $\mathrm{a}=69.7, \mathrm{~A}=0.8795+0.0459 \exp \left(-\left(\lambda_{\max }-300\right)^{2} / 11940\right)$, $\mathrm{b}=28, \mathrm{~B}=0.922, \mathrm{c}=-14.9, \mathrm{C}=1.104, \mathrm{D}=0.674, \mathrm{~A}_{\beta}=0.26, \lambda_{\beta}=189+0.315 \lambda_{\max }$, and $\mathrm{d}=-$ $40.5+0.195 \lambda_{\max }$

\section{Self-screening}

The capacity of photoreceptors to absorb light can be described in terms of its photopigment optical density due to a process known as 'self-screening'. ${ }^{56}$ The self-screening is corrected for by modifying the photopigment absorption spectrum from the normalized low density absorbance $\mathrm{S}_{\mathrm{q}, \mathrm{D}}(\lambda)$ as

$$
S_{q, P}(\lambda)=1-10^{-D_{\text {peak }} \times S_{q, D}(\lambda)},
$$

where $D_{\text {peak }}$ is the assumed peak optical density. ${ }^{49}$

Changes in optical density do not affect the pigment peak sensitivity, but they do change its spectral absorbance. A cell with a higher optical density of photopigments will also be more sensitive across the spectrum. As a result, the spectral sensitivity curve of a photoreceptor is broader than that of a single photopigment. Two photoreceptors expressing the same photopigment at different optical densities will, therefore, have different spectral sensitivities.

\section{Pre-retinal filtering}

Pre-retinal filtering describes filtering of the light as is passes through the lens and other preretinal optical media in the eye. The optical density of the eye media of an average observer can be estimated as a function of age $A$. Here we use the age-dependent human ocular media model of van de Kraats and van Norren, ${ }^{37}$ which is written as a sum of five spectral components and a spectrally neutral offset

$$
\begin{aligned}
& D_{\mathrm{M}}(\lambda, A)=d_{\mathrm{RL}}(A) \times M_{\mathrm{RL}}(\lambda)+M_{\mathrm{TP}}(\lambda)+d_{\mathrm{LY}}(A) \times M_{\mathrm{LY}}(\lambda)+d_{\mathrm{LOUV}}(A) \times \\
& M_{\mathrm{LOUV}}(\lambda)+d_{\mathrm{LO}}(A) \times M_{\mathrm{LO}}(\lambda)+d_{\text {neutral }},
\end{aligned}
$$

where $M$ are the templates for describing the spectral shape of each component and $d$ are agedependent scalar weights (the density coefficients). ${ }^{51}$ The subscripts RL, TP, LY, LOUV, and LO are Rayleigh loss, tryptophan, lens young, lens old UV, and lens old, respectively. Optical density is expressed as the negative log of the transmission. Thus the spectral transmittance for age $A$ is expressed as

$$
\tau(\lambda, A)=10^{-D_{M}(\lambda, A)} \text {. }
$$

\section{Corneal spectral sensitivity}

Finally the corneal spectral sensitivity is acquired by 


$$
S_{\mathrm{q}, \mathrm{C}}=S_{\mathrm{q}, \mathrm{P}} \times \tau(\lambda, A)
$$

and then converted from units of quanta to energy at each wavelength. The curve is converted to energy units by multiplying by wavelength number $\lambda$ and then normalized. This procedure results in a shift of peak wavelength and a change in the shape of the spectral curve. For example, the sensitivity of ipRGCs constructed at the corneal plane peaks at $490 \mathrm{~nm}$, while the maximum absorbance wavelength was set to $480 \mathrm{~nm}$, and the short wavelength 'tail' of the ipRGCs spectral function is lowered due to pre-retinal filtering thus the response below $400 \mathrm{~nm}$ falls toward zero. 